\title{
Nucleation and propagation of phase mixtures in a bistable chain
}

\author{
Anna Vainchtein* \\ Department of Mathematics, University of Pittsburgh, Pittsburgh, Pennsylvania 15260, USA
}

Erik S. Van Vleck ${ }^{\dagger}$

Department of Mathematics, University of Kansas, Lawrence, Kansas 66045, USA

(Received 15 February 2009; revised manuscript received 26 March 2009; published 29 April 2009)

\begin{abstract}
We consider a prototypical discrete model of phase transitions. The model consists of a chain of particles, each interacting with its nearest and next-to-nearest neighbors. The long-range interaction between next-tonearest neighbors is assumed to be harmonic, while the nearest-neighbor interactions are nonlinear and bistable. We consider overdamped dynamics of the chain and after suitable rescaling obtain a discrete reactiondiffusion equation with a negative diffusion coefficient. Using a biquadratic nearest-neighbor interaction potential and introducing new variables, we construct and study traveling-wave-like solutions that describe dynamics of phase mixtures in the lattice. Depending on the value of the applied force, phase mixtures either get trapped in one of the multiple equilibrium states or propagate through the chain at a constant speed. At low velocities near the depinning threshold, the motion is of stick-slip type. Numerical results for smoother potentials also suggest the existence and stability of the steady motion in a certain range of applied loads.
\end{abstract}

DOI: 10.1103/PhysRevB.79.144123

PACS number(s): 64.70.K-, 61.72.Bb, 63.90.+t

\section{INTRODUCTION}

Materials undergoing martensitic phase transitions often form finely layered twinning microstrutures. ${ }^{1,2}$ The propagation of fine mixtures of two different martensitic variants inside the parent austenite phase is responsible for hysteretic behavior exhibited by these materials under cyclic loading. Much of recent work has focused on the kinetics of an isolated phase boundary, but when a fine microstructure is present, the interaction between the phase boundaries cannot be ignored, and dynamics of a phase mixture must be described as a whole.

In this paper we study a prototypical discrete model that accounts for nucleation and propagation of phase mixtures under applied loading. Specifically, we consider a chain of particles, each interacting with its nearest and next-to-nearest neighbors (NNs). To model a phase transition, we assume that the nearest-neighbor interactions are described by a nonlinear nonconvex interaction potential with two convex regions representing two different material phases or variants. To mimic the effect of higher dimensions, we also include harmonic next-to-nearest-neighbor (NNN) interactions. Together with the boundary conditions, these long-range bonds bring in an additional constraint that models the presence in these materials of the parent austenite phase that is incompatible with a single martensite variant and forces the formation of finely layered microstructures. As we shall see, the effect of the harmonic next-to-nearest interactions is to induce fine-scale oscillations of elastic strain between the two phases and thus form a phase mixture. In essence, this model represents a nonlocal discrete analog of the one-dimensional continuum models of a phase-transforming bar on an elastic foundation. $^{3-5}$ From the calculus of variations point of view, these models were motivated by a desire to mimic in a onedimensional setting the lack of lower semicontinuity of the nonconvex free energy in higher dimensions that leads to the formation of finer and finer microstructures. ${ }^{6,7}$ Unlike the continuum models mentioned above, where the microstruc- tures can become infinitely fine, the present discrete model has an inherent length scale, the distance between neighboring particles, which determines the scale of the microstructure. In the continuum setting, attempts have been made to account for this effect of discreteness by including an oscillation-inhibiting strain-gradient term in the free energy ${ }^{8-11}$ but such models do not capture the behavior of the discrete system near the quasistatic limit. ${ }^{12}$

As shown in Ref. 13, the discrete model considered here can also be derived from a lattice model that simulates shear in a two-dimensional body. In Ref. 13 the equilibrium states of a finite chain under displacement-controlled loading were analyzed, and the continuum limit was obtained via $\Gamma$ convergence. In this paper we study the dynamics of the phase mixtures. With this in mind we consider an infinite chain and account for microlevel dissipation due to other degrees of freedom by assuming that the nearest-neighbor bonds are viscoelastic. To focus on the small-velocity region of phase mixture kinetics where inertia can be neglected, we consider the overdamped dynamic limit. The resulting dynamical system reduces to what could be referred to as a discrete Nagumo equation but with "negative diffusion." We consider both numerical simulations and analytical solutions derived for the case of a bilinear nonlinearity ${ }^{14,15}$ using transform techniques after writing the problem as a system in terms of the odd-numbered and even-numbered lattice variables. We uncover a rich structure of solution behavior that includes trapped equilibrium solution, single-front solutions, and solutions composed of two fronts moving apart. We also consider stability of solutions and further illustrate the behavior of solutions with trilinear and cubic nonlinearities.

There has been much work on traveling-wave solutions of the discrete Nagumo equations, which in addition to biological applications, also arise as the overdamped limit of the Frenkel-Kontorova model, widely used in physics to model friction, dislocation motion, charge-density waves, and other phenomena. ${ }^{16}$ In one dimension the equation usually represents an infinite chain of particles with nearest-neighbor har- 
monic coupling placed in a bistable nonlinear potential, where the coupling, or diffusion, constant is positive. Among the papers studying this problem are Refs. 17-19. The important difference between this model and the one studied here is that the "negative diffusion" problem we study involves the formation and motion of multiple interfaces that form microstructures, while the positive-diffusion problems involve a single front. In Ref. 20 a discrete Nagumo equation over a two-dimensional lattice is considered, with the focus on the behavior of plane-wave solutions and their dependence of the direction of propagation when the nonlinearity is bilinear. The case of negative coupling constant studied here is also briefly mentioned in Ref. 20, and in Ref. 21-23 it is shown that in two dimensions it leads to various stripe and checkerboard patterns.

We remark that in statics the discrete model studied here can be characterized as an antiferromagnetic Ising model (e.g., Ref. 24) with elastic wells. Indeed, the nonconvex potential, describing nearest-neighbor interactions generates a spinlike variable, while the harmonic potential for next-tonearest neighbors ensures that these "spins" interact. If the quadratic wells are replaced by the infinitely steep walls located at \pm 1 , the static problem reduces exactly to the antiferromagnetic Ising model. We note also that the extension of the antiferromagnetic Ising model (ANNNI model) and the Frenkel-Kontorova-type models that incorporate competing interactions have been used to study transitions between high-symmetry, commensurate, and incommensurate phases in crystals, ${ }^{25,26}$ with the focus mostly on the ground states as well as numerical simulations and continuum approximation of the dynamics in the absence of applied load. In this context, we derive here the exact kinetics of the domain walls separating commensurate and high-symmetry (homogeneous) phases under applied load. To allow for the possibility of the transition to the incommensurate phase, one needs to include competing long-range interactions; the study of the corresponding dynamics is left to the future investigations.

This paper is organized as follows. In Sec. II we derive the model to be considered starting from an infinite chain of particles with nearest- and next-to-nearest-neighbor interactions. The model that we obtain reduces to a spatially discrete reaction-diffusion equation but with a negative diffusion coefficient. In Sec. III, numerical simulations give an indication of the solution behavior. In Sec. IV we derive analytical solutions using transform techniques and further investigate their stability properties. These include one-front solutions, two-front solutions in which two fronts are moving apart, and trapped equilibrium states that exist between certain threshold values of the applied load. We investigate the kinetic relation and observe good agreement between the numerical and analytically obtained solutions. Further numerical simulations are performed using the trilinear and cubic nonlinearities in Sec. V. The results are summarized and discussed in Sec. VI.

\section{MODEL}

Consider a chain of particles, each interacting with its nearest and next-to-nearest neighbors, as shown in Fig. 1.

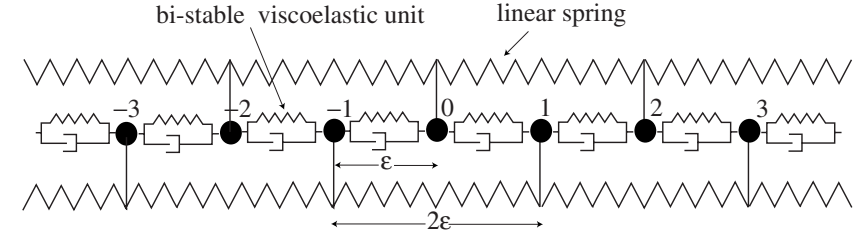

FIG. 1. One-dimensional chain with viscoelastic nearest- and elastic next-to-nearest-neighbor interactions.

For simplicity we assume that the long-range interaction between next-to-nearest neighbors is harmonic, with elastic modulus $\gamma>0$, while the nearest-neighbor interactions are nonlinear, with elastic energy density $h(w)$. If $u_{n}(t)$ is the displacement of the $n$th particle, the sum of kinetic and internal energies of the chain can then be written as

$$
\mathcal{E}=\varepsilon \sum_{n=-\infty}^{\infty}\left[\frac{\rho \dot{u}_{n}^{2}}{2}+h\left(\frac{u_{n+1}-u_{n}}{\varepsilon}\right)+\gamma\left(\frac{u_{n+2}-u_{n}}{\varepsilon}\right)^{2}\right],
$$

where $\varepsilon$ is the reference interparticle distance, $\rho$ is the density of the chain per unit length, and $\dot{u}_{n}(t) \equiv u_{n}^{\prime}(t)$ is the velocity of $n$th particle. Note that internal energy of a uniformly deformed chain $\left(u_{n}=w n \varepsilon\right.$ for all $\left.n\right)$ is given by $\mathcal{W}$ $=\varepsilon\left[h(w)+4 \gamma w^{2}\right]$. Hence the macroscopic elastic modulus of the chain in the undeformed configuration $(w=0)$ equals

$$
E=h^{\prime \prime}(0)+4 \gamma,
$$

and in order to ensure stability of the undeformed chain we must require that $E>0$.

To model phase transitions in the chain, we select a nonconvex NN interaction potential $h(w)$, with two convex regions representing two different phases. In order to obtain explicit solutions we will assume that the potential is biquadratic,

$$
h(w)= \begin{cases}\frac{1}{2} K w^{2}, & w \leq w_{c} \\ \frac{1}{2} K(w-a)^{2}+K a\left(w_{c}-\frac{a}{2}\right), & w \geq w_{c}\end{cases}
$$

see Fig. 2. Thus, the NN springs can exist in two different states (phases), depending on whether their elastic strain,

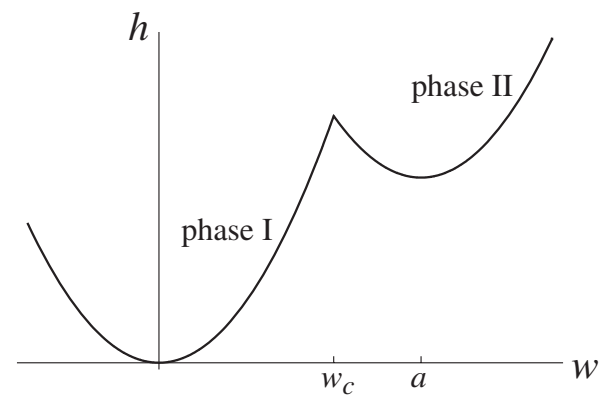

FIG. 2. The biquadratic NN interaction potential [Eq. (3)]. 


$$
w_{n}=\frac{u_{n}-u_{n-1}}{\varepsilon},
$$

is below (phase I) or above (phase II) the critical strain $w_{c}$. Here $K>0$ is the elastic modulus in each phase, and $a>0$ is the microscopic transformation strain, a parameter generally independent of $w_{c}$. With this assumption, the macroscopic elastic modulus [Eq. (2)] becomes $E=K+4 \gamma>0$. The last inequality together with $K>0$ ensures stability of the homogeneously deformed chain at noncritical strain. ${ }^{27}$ It is also convenient to introduce macroscopic transformation strain,

$$
\Delta=\frac{a K}{E} .
$$

Assume further that the nearest-neighbor interactions are viscoelastic, with the total interaction force given by $f(w, \dot{w})=h^{\prime}(w)+\xi \dot{w}$. Here $\xi>0$ is the viscosity coefficient. The dynamics of the chain with energy [Eq. (1)] is then governed by the following infinite system of ordinary differential equations:

$$
\begin{aligned}
\rho \ddot{u}_{n}= & \frac{1}{\varepsilon}\left[h^{\prime}\left(\frac{u_{n+1}-u_{n}}{\varepsilon}\right)-h^{\prime}\left(\frac{u_{n}-u_{n-1}}{\varepsilon}\right)\right. \\
& \left.+\frac{\gamma}{\varepsilon}\left(u_{n+2}-2 u_{n}+u_{n-2}\right)+\frac{\xi}{\varepsilon}\left(\dot{u}_{n+1}-2 \dot{u}_{n}+\dot{u}_{n-1}\right)\right] .
\end{aligned}
$$

There are two time scales associated with this problem: the time scale of inertia, $T_{\mathrm{in}}=\varepsilon \sqrt{\rho / E}$, and the viscosity time scale, $T_{\text {visc }}=\xi / E$. In this paper we will consider an overdamped chain in which $T_{\text {visc }} \gg T_{\text {in }}$, i.e., we assume that

$$
\xi \gg \varepsilon \sqrt{\rho E} \text {. }
$$

Using $T_{\text {visc }}$ as the time scale, $\varepsilon$ as the length scale and the macroscopic modulus $E$ as the force scale, we introduce the following dimensionless variables:

$$
\bar{t}=\frac{t}{T_{\text {visc }}}, \quad \bar{u}_{n}=\frac{u_{n}}{\Delta \varepsilon}, \quad \bar{w}_{n}=\frac{w_{n}}{\Delta}, \quad \bar{w}_{c}=\frac{w_{c}}{\Delta}, \quad \bar{h}=\frac{h}{E \Delta^{2}} .
$$

Here we use macroscopic transformation strain [Eq. (4)] to rescale strains for convenience. Dropping the bars on the new variables and neglecting inertia term in view of Eq. (6), we obtain the following dimensionless equation in terms of strain variables:

$$
\begin{aligned}
\dot{w}_{n+1}-\dot{w}_{n}= & -\alpha\left(w_{n+2}+w_{n+1}-w_{n}-w_{n-1}\right)+h^{\prime}\left(w_{n}\right) \\
& -h^{\prime}\left(w_{n+1}\right),
\end{aligned}
$$

where

$$
\alpha=\frac{\gamma}{E}
$$

is the parameter measuring the relative strength of NNN interactions. Note that due to positive $\gamma$ and $K$ we have (recall that $E=K+4 \gamma$ )

$$
0<\alpha<0.25
$$

Observe that Eq. (8) can be "integrated" to yield

$$
\dot{w}_{n}=-\alpha\left(w_{n+1}-2 w_{n}+w_{n-1}\right)-\sigma\left(w_{n}\right)+\sigma_{a},
$$

where

$$
\sigma(w)=h^{\prime}(w)+4 \alpha w
$$

is the macroscopic stress-strain law in the chain, and $\sigma_{a}$ is the constant of integration that has the physical meaning of applied stress. For biquadratic NN interactions [Eq. (3)] we have bilinear stress-strain relation,

$$
\sigma(w)=w-\theta\left(w-w_{c}\right),
$$

where $\theta(w)$ is a unit step function; in general $\sigma(w)$ is a nonmonotone up-down-up function of strain. One can see that Eq. (11) is a spatially discrete reaction-diffusion equation with a negative diffusion coefficient $D=-\alpha$. Observe that the (dimensionless) free energy of the chain can be written as

$$
\mathcal{G}=\sum_{n=-\infty}^{\infty}\left[H\left(w_{n}\right)-\alpha\left(w_{n}-w_{n+1}\right)^{2}-\sigma_{a} w_{n}\right]
$$

where

$$
H(w)=h(w)+2 \alpha w^{2}= \begin{cases}\frac{1}{2} w^{2}, & w \leq w_{c} \\ \frac{1}{2}(w-1)^{2}+w_{c}-\frac{1}{2}, & w \geq w_{c}\end{cases}
$$

is the macroscopic energy density [note that $\sigma(w)=H^{\prime}(w)$ ]. The first term in the free energy induces phase transitions, and the second term is the discrete analog of a higher-order strain-gradient term in the total energy. Clearly, negative $\alpha$ (repulsive NNN interactions, positive diffusion coefficient) penalizes oscillations in $w_{n}$ and hence results in fewer phase boundaries. In contrast, positive $\alpha$ considered here favors the formation of phase mixtures. ${ }^{13}$ The dynamics of these phase mixtures is the subject of this paper.

\section{NUMERICAL EXPERIMENTS}

To get an idea of how the phase mixtures form and propagate, we solved Eq. (11) numerically using NDSolve routine in MATHEMATICA. As the initial condition, we chose an equilibrium state in phase $\mathrm{I}, w_{n}=\sigma_{a}<w_{c}$, perturbed by setting a single spring in the middle of the chain to have critical strain $w_{c}$. The boundary conditions require that $w_{n}=w_{n+1}$ at either end of the chain.

At applied stress $\sigma_{a}$ below a certain threshold $\sigma_{a}^{t r}$ dependent on $\alpha$, there is no propagation of phase boundaries. The spring initially set at critical strain transforms to phase II, and the neighboring springs adjust their strains (still in phase I) accordingly. After that the solution remains in a "trapped" stationary state such as the ones depicted in Fig. 3. At applied stress above the threshold, this equilibrium state becomes unstable, and a fine mixture of two phases forms. After an initial transient period, it propagates steadily along the chain (see Fig. 4). In the phase mixture each two neigh- 

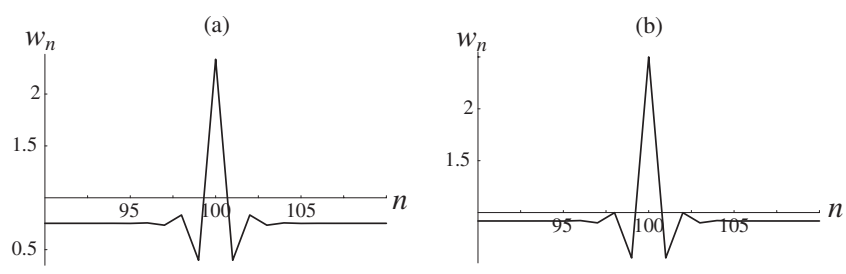

FIG. 3. The trapped equilibrium states approached by the dynamic solution at applied stress $\sigma_{a}<\sigma_{a}^{t r}$ : (a) $\sigma_{a}=0.754$; (b) $\sigma_{a}$ $=0.919$. Parameters: $w_{c}=1, \alpha=0.15$

boring points are in different phases. Note that the phase mixture has clearly defined maximum and minimum strain values that stay constant as the mixture propagates along the chain.

\section{ANALYTICAL SOLUTION}

In this section we construct explicit solutions for the dynamics of phase mixtures.

\section{A. Propagation of phase mixtures: A single front}

Observing that the period of strain oscillations in a phase mixture equals the lattice period, we consider the evolution of even-numbered and odd-numbered springs. Specifically, we introduce new variables $y_{n}=w_{2 n}$ (even-numbered strains) and $z_{n}=w_{2 n+1}$ (odd-numbered). Using Eq. (11), we obtain the system of equations governing evolution of the new variables,
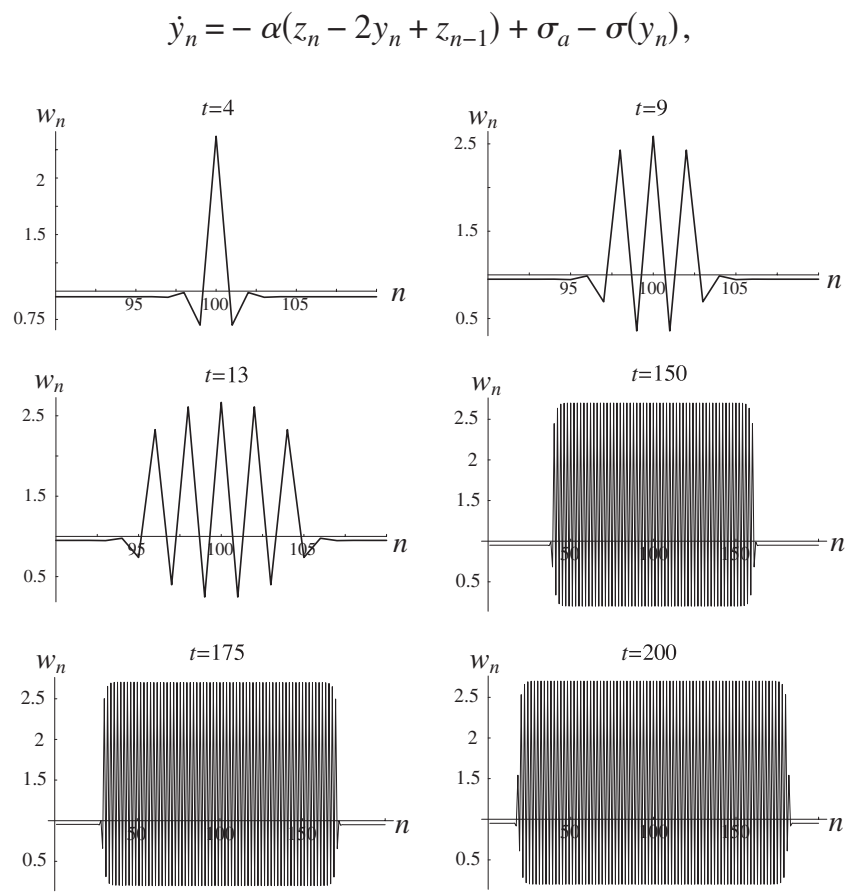

FIG. 4. Evolution of strain above the threshold applied stress. Parameters: $\sigma_{a}=0.95, \alpha=0.15, w_{c}=1$.

$$
\dot{z}_{n}=-\alpha\left(y_{n}-2 z_{n}+y_{n+1}\right)+\sigma_{a}-\sigma\left(z_{n}\right) .
$$

In this section we focus on a single front of the moving phase mixture. We thus seek solution of Eq. (16) in the form of a traveling wave moving with velocity $c$,

$$
y_{n}(t)=\phi(\xi)+\sigma_{a}, \quad z_{n}(t)=\psi(\xi)+\sigma_{a}, \quad \xi=n-c t .
$$

Note that this means that the actual velocity of the phase mixture is $V=2 c$, since $y_{n}(t)=w_{2 n}(t)$ by definition. We will assume that at $\xi<0(n<c t)$ the even-numbered strains are in phase II: $y_{n}(t)>w_{c}$, or $\phi(\xi)>\phi_{c}$, where

$$
\phi_{c}=w_{c}-\sigma_{a},
$$

while at $\xi>0$ the even-numbered strains are in phase I $\left(y_{n}(t)<w_{c}\right.$, or $\left.\phi(\xi)<\phi_{c}\right)$. Meanwhile, the odd-numbered strains remain in phase $\mathrm{I}\left(z_{n}(t)<w_{c}\right)$ for all $\xi$. Using these assumptions along with Eqs. (13), (16), and (17), we obtain the following system of two advance-delay differential equations:

$$
\begin{gathered}
-c \phi^{\prime}(\xi)=-\alpha[\psi(\xi)-2 \phi(\xi)+\psi(\xi-1)]-\phi(\xi)+\theta(-\xi), \\
-c \psi^{\prime}(\xi)=-\alpha[\phi(\xi)-2 \psi(\xi)+\phi(\xi+1)]-\psi(\xi) .
\end{gathered}
$$

In addition, we impose the following conditions at infinity:

$$
\begin{gathered}
\phi(+\infty)=\psi(+\infty)=0, \\
\phi(-\infty)=\phi_{-}, \quad \psi(-\infty)=\psi_{-},
\end{gathered}
$$

with $\phi_{-}$and $\psi_{-}$to be determined. Finally, for consistency with the assumptions used to obtain Eq. (19), we must require that the even-numbered strains switch phase at $\xi=0$,

$$
\phi(0)=\phi_{c},
$$

with $\phi_{c}$ defined in Eq. (18), and that the inequalities

$$
\begin{gathered}
\phi(\xi)>\phi_{c}, \quad \xi<0 \quad \text { and } \quad \phi(\xi)<\phi_{c}, \quad \xi>0, \\
\psi(\xi)<\phi_{c} \text { for all } \xi
\end{gathered}
$$

hold. We seek solutions of Eq. (19) subject to Eqs. (20)-(23). Applying the generalized Fourier transform,

$$
\hat{\phi}(k)=\int_{-\infty}^{\infty} \phi(k) e^{-i \xi k} d \xi,
$$

to Eq. (19), solving for resulting algebraic system for $\hat{\phi}(k)$, $\hat{\psi}(k)$ and taking the inverse transform,

$$
\phi(\xi)=\frac{1}{2 \pi} \int_{-\infty}^{\infty} \hat{\phi}(\xi) e^{i \xi k} d k
$$

we obtain

$$
\phi(\xi)=\frac{1}{2 \pi i} \int_{\Gamma} \frac{i c k+2 \alpha-1}{\left[(i c k+2 \alpha-1)^{2}-4 \alpha^{2} \cos ^{2}(k / 2)\right] k} e^{i k \xi} d k,
$$




$$
\psi(\xi)=\frac{\alpha}{\pi i} \int_{\Gamma} \frac{\cos (k / 2)}{\left[(i c k+2 \alpha-1)^{2}-4 \alpha^{2} \cos ^{2}(k / 2)\right] k} e^{i k(\xi+1 / 2)} d k
$$

where the contour $\Gamma$ coincides with the real axis everywhere except near the point $k=0$, where the integrands are singular. To resolve the singularity in accordance with the conditions (20), the contour goes above $k=0$. Using the residue theorem, we then obtain

$$
\phi(\xi)= \begin{cases}\frac{1-2 \alpha}{1-4 \alpha}-\sum_{k \in M^{-}(c)} \frac{i c k+2 \alpha-1}{k \mathcal{L}_{k}(k, c)} e^{i k \xi}, & \xi<0 \\ \sum_{k \in M^{+}(c)} \frac{i c k+2 \alpha-1}{k \mathcal{L}_{k}(k, c)} e^{i k \xi}, & \xi>0\end{cases}
$$

and

$\psi(\xi)$

$$
= \begin{cases}-\frac{2 \alpha}{1-4 \alpha}-2 \alpha \sum_{k \in M^{-}(c)} \frac{\cos (k / 2)}{k \mathcal{L}_{k}(k, c)} e^{i k(\xi+1 / 2)}, & \xi<-1 / 2 \\ 2 \alpha \sum_{k \in M^{+}(c)} \frac{\cos (k / 2)}{k \mathcal{L}_{k}(k, c)} e^{i k(\xi+1 / 2)}, & \xi>-1 / 2 .\end{cases}
$$

Here we defined the characteristic function

$$
\mathcal{L}(k, c)=(i c k+2 \alpha-1)^{2}-4 \alpha^{2} \cos ^{2}(k / 2),
$$

$\mathcal{L}_{k} \equiv \partial \mathcal{L} / \partial k$, and the sums in Eqs. (25) and (26) are over the roots of $\mathcal{L}(k, c)=0$,

$$
M^{ \pm}(c)=\{k \mid \mathcal{L}(k, c)=0, \operatorname{Im} k \gtrless 0\} .
$$

Note that Eqs. (25) and (26) imply that $\phi_{-}$and $\psi_{-}$in Eq. (20) are given by $\phi_{-}=(1-2 \alpha) /(1-4 \alpha)$ and $\psi_{-}=-2 \alpha /(1-4 \alpha)$. Since $0<\alpha<0.25$, we have $\phi_{-}>0$ and $\psi_{-}<0$, which is consistent with our assumption that even-numbered strains $y_{n}$ decrease from phase II to phase I values across the front, while the odd-numbered strains remain in phase I.

The problem thus reduces to finding the roots of the characteristic function (27). First, observe that at $c>0$ there is at least one purely imaginary root $k=i r$, where $r$ satisfies the transcendental equation

$$
(r c+1-2 \alpha)^{2}=4 \alpha^{2} \cosh ^{2}(r / 2),
$$

which can be solved numerically. Indeed, the parabola in the left-hand side, which has zero minimum at $r=2 \alpha-1<0$ (recall that $0<\alpha<0.25$ ) always has at least one intersection with the function on the right-hand side, which is bounded by $4 \alpha^{2}>0$ from below, implying the existence of at least one solution. The other roots of $\mathcal{L}(k, c)=0$ can be found by noting that at $c=0$ and $\alpha<0.25$ the set of roots is given by

$$
k=2 \pi n \pm i \rho, \quad n=0,1, \ldots,
$$

where

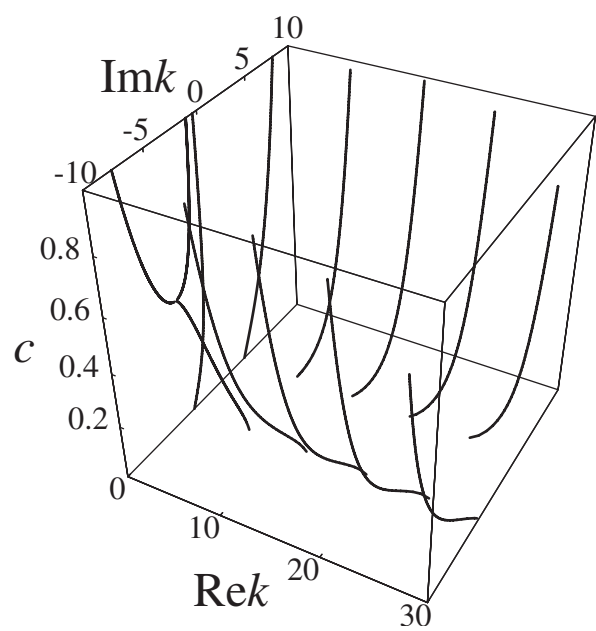

FIG. 5. Roots of $\mathcal{L}(k, c)=0$ at $\alpha=0.15$. Due to symmetry of roots about the imaginary axis, only roots with positive real parts are shown.

$$
\rho=4 \operatorname{arccosh} \frac{1}{2 \sqrt{\alpha}}=\operatorname{arccosh} \frac{2 \alpha^{2}-4 \alpha+1}{2 \alpha^{2}} .
$$

Note that $\rho$ is a real number since $0<\alpha<0.25$.

Using these roots as initial guesses, we can find numerically the roots at small nonzero $c$. Continuing the procedure, we find all roots along the branches emanating from Eq. (29). The results for $\alpha=0.15$ are shown in Fig. 5.

To complete the solution, we need to impose the phase switch condition (21) on solution (25) for $\phi(\xi)$. In view of Eq. (18), this yields the following relation between applied stress $\sigma_{a}$ and the velocity $V=2 c$ of the phase mixture front,

$$
\sigma_{a}=\sigma_{M}+\bar{\sigma}(V),
$$

where $\sigma_{M}=w_{c}-1 / 2$ is the Maxwell stress, the stress that divides the curve $\sigma(w)$ into two equal areas, and

$$
\begin{aligned}
\bar{\sigma} & =\frac{1}{2}-\frac{1-2 \alpha}{1-4 \alpha}+\sum_{k \in M^{-}(V / 2)} \frac{i V k / 2+2 \alpha-1}{k \mathcal{L}_{k}(k, V / 2)} \\
& =\frac{1}{2}-\sum_{k \in M^{+}(V / 2)} \frac{i V k / 2+2 \alpha-1}{k \mathcal{L}_{k}(k, V / 2)} .
\end{aligned}
$$

Here the second equality follows from continuity of Eq. (25) at $\xi=0$. Thus, for a given $V$, one can compute the applied stress $\sigma_{a}$ from Eqs. (31) and (32), furnishing a kinetic relation. Substituting it in Eqs. (25) and (26) and recalling Eq. (17), we obtain the solution for all (even and odd-numbered) strains.

\section{B. Stability of one-front solutions}

The standard approach to determining stability properties of traveling-wave solutions to partial differential equations is to employ a moving coordinate system so that the problem becomes essentially one of determining the stability of a steady-state solution. In the case of lattice differential equations this approach is difficult to follow. In particular, introducing $\xi=n-c t$ and letting $w_{n}(t)=v(t, n-c t)$ transforms Eq. 
(11) into the advance-delay partial differential equation,

$$
\begin{aligned}
v_{t}(t, \xi)-c v_{\xi}(t, \xi)= & -\alpha[v(t, \xi-1)-2 v(t, \xi)+v(t, \xi+1)] \\
& -\sigma[v(t, \xi)]+\sigma_{a}
\end{aligned}
$$

about which very little is known. Instead we employ an alternative approach following the work of Chow, MalletParet, and Shen. ${ }^{28}$ Let $p_{n}(t)$ denote the traveling-wave solution obtained in Eqs. (25) and (26), i.e., $p_{n}(t)=\phi(n-c t)$ for $n$ even and $p_{n}(t)=\psi(n-c t)$ for $n$ odd. Linearizing about this solution, we obtain

$$
\dot{u}_{n}=-\alpha\left(u_{n-1}-2 u_{n}+u_{n+1}\right)-u_{n}-g_{n}(t) u_{n}(t),
$$

where $g_{n}(t)=\delta_{0}(n-c t)$, the Dirac delta function, for $n$ even, and $g_{n}(t)=0$ for $n$ odd. Notice that the traveling-wave solution satisfies $p_{n}(t)=p_{n+2}(t+2 / c)$, so the traveling-wave solution can be viewed as a periodic orbit (modulo a shift in $n$ ) of period $2 / c$. This is the approach taken in Ref. 28. For the equation $\dot{v}=F(v)$ with traveling-wave solution $p(t)$ and wave speed $c$ we linearize about $p$ and consider $\dot{u}=F^{\prime}(p) u$, or $\frac{d}{d t}\left(S^{2} u\right)=S^{2} F^{\prime}(p) S^{-2}\left(S^{2} u\right)$, where $S$ is the shift operator such that $(S u)_{i}=u_{i+1}$ and $\left(S^{-1} u\right)_{i}=u_{i-1}$. Then for $\Gamma(t): l^{\infty}(\mathbb{Z})$ $\rightarrow l^{\infty}(\mathbb{Z})$, a fundamental solution matrix of the linearized problem, $S^{2} \Gamma(2 / c) \dot{p}(0)=S^{2} \dot{p}(2 / c)=\dot{p}(0)$ so that $\lambda=1$ is an eigenvalue of the operator $S^{2} \Gamma(2 / c)$ with associated eigenvector $\dot{p}(0)$.

Consider a wave speed $c>0$. We use transform techniques and modified Bessel functions of the first kind to construct the action of the operator $\Gamma$. Instead of $t$ $\in[0,2 / c]$, we consider $t \in[-1 / c, 1 / c]$ and note that in this range of $t, \quad g_{n}(t)=0$ unless $n=0$. Thus, for $\hat{u}(k, t)$ $=\sum_{n} e^{i k n} u_{n}(t)$ and $t \in[-1 / c, 1 / c]$,

$$
\frac{d}{d t} \hat{u}(k, t)=\nu(k) \hat{u}(k, t)-g_{0}(t) u_{0}(t)
$$

where $\nu(k)=-2 \alpha[\cos (k)-1]-1$. By the variation in parameters formula,

$$
\hat{u}(k, t)=e^{\nu(k) t}\left[\hat{u}(k, 0)-\int_{0}^{t} e^{-\nu(k) s} g_{0}(s) u_{0}(s) d s\right]
$$

and

$$
\begin{aligned}
u_{n}(t)= & \frac{1}{2 \pi} \int_{-\pi}^{\pi} e^{-i k n} \hat{u}(k, t) d k=\frac{1}{2 \pi} \int_{-\pi}^{\pi} e^{-i k n} e^{\nu(k) t} \\
& \times\left[\hat{u}(k, 0)-t \int_{0}^{t} e^{-\nu(k) s} g_{0}(s) u_{0}(s) d s\right] d k .
\end{aligned}
$$

We next focus on evaluating this expression at $t= \pm 1 / \mathrm{c}$ and observe that due to the standard properties of the delta function we have

$$
\int_{0}^{ \pm 1 / c} e^{-\nu(k) s} g_{0}(s) u_{0}(s) d s= \pm \frac{1}{2 c} u_{0}(0) .
$$

Then

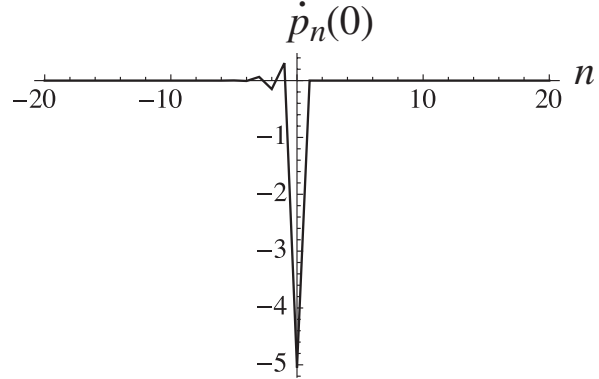

FIG. 6. Plot of $\dot{p}(0)$ for $\alpha=0.15, w_{c}=1$, and $V=0.2$.

$u_{n}( \pm 1 / c)=\frac{1}{2 \pi} \int_{-\pi}^{\pi} e^{-i k n} e^{ \pm \nu(k) / c}\left[\sum_{m} e^{i k m} u_{m}(0) \mp \frac{1}{2 c} u_{0}(0)\right] d k$

or

$$
\begin{aligned}
u_{n}( \pm 1 / c)= & \frac{1}{2 \pi} \int_{-\pi}^{\pi} e^{-i k n} e^{ \pm \nu(k) / c} \\
& \times\left[\left(1 \mp \frac{1}{2 c}\right) u_{0}(0)+\sum_{m \neq 0} e^{i k m} u_{m}(0)\right] d k
\end{aligned}
$$

We make use of the following identity in terms of modified Bessel functions of the first kind, $I_{j}(x)$ :

$$
\frac{1}{2 \pi} \int_{-\pi}^{\pi} e^{i j k} e^{ \pm \nu(k) / c} d k= \pm(-1)^{j} e^{ \pm(2 \alpha-1) / c} I_{j}(2 \alpha / c),
$$

to obtain

$$
\begin{aligned}
u_{n}( \pm 1 / c)= & \pm e^{ \pm(2 \alpha-1) / c}\left[\left(1 \mp \frac{1}{2 c}\right) u_{0}(0)(-1)^{n} I_{n}(2 \alpha / c)\right. \\
& \left.+\sum_{m \neq 0}(-1)^{n-m} u_{m}(0) I_{n-m}(2 \alpha / c)\right]
\end{aligned}
$$

If $u(0)=\dot{p}(0)$, then $\left.\|u(-1 / c)\|\right|_{l^{\infty}(\mathrm{Z})}=\left.\|u(1 / c)\|\right|_{l^{\infty}(\mathrm{Z})}$ since $S^{2} u(1 / c)=u(-1 / c)$. Since $\dot{p}(0) \in l^{\infty}(\mathbb{Z})$ and $\dot{p}(0) \in l^{2}(\mathbb{Z})$, we can find a spanning set for the complement to $\dot{p}(0)$ in $l^{\infty}(\mathbb{Z})$ by employing

$$
u^{(j)}(0)=e_{j}-\frac{\left\langle e_{j}, \dot{p}(0)\right\rangle}{\langle\dot{p}(0), \dot{p}(0)\rangle} \dot{p}(0) \equiv e_{j}-\beta_{j} \dot{p}(0)
$$

for $j \in \mathbb{Z}$, where $\langle x, y\rangle$ is the standard inner product in $l^{2}(\mathbb{Z})$. We are able to infer stability of the one-front solution using the results in Ref. 28 if $\left.\left\|u^{(j)}(-1 / c)\right\|\right|_{l^{\infty}(\mathrm{Z})}>\left.\left\|u^{(j)}(1 / c)\right\|\right|_{l^{\infty}(\mathrm{Z})}$ for $j \in \mathbb{Z}$.

We tested this numerically for $\alpha=0.15, w_{c}=1$, and $V$ $=0.1,0.2$, and 0.4. First note that due to the fact that the support of $\dot{p}(0)$ (see Fig. 6) is centered near $n=0, \beta_{j} \rightarrow 0$ as $|j| \rightarrow \infty$. Writing $u_{n}[ \pm 1 / c ; u(0)]$ as the solution in Eq. (34) with initial condition $u(0)$ we have

$$
\begin{aligned}
\left|u_{n}\left(-1 / c ; e_{j}\right)\right| & =e^{-(2 \alpha-1) / c} I_{n-j}(2 \alpha / c)>e^{(2 \alpha-1) / c} I_{n-j}(2 \alpha / c) \\
& =\left|u_{n}\left(1 / c ; e_{j}\right)\right|
\end{aligned}
$$

since $2 \alpha-1<0$ and $c>0$. We computed $u[1 / c ; \dot{p}(0)]$, 
formed $u[-1 / c ; \dot{p}(0)]=S^{2} u[1 / c ; \dot{p}(0)]$ and found $\beta_{j}$ as in Eq. (35). We found that

$$
\begin{aligned}
\sup _{n}\left|u_{n}\left(-1 / c ; e_{j}\right)-\beta_{j} u_{n}[-1 / c ; \dot{p}(0)]\right|>\sup _{n} \mid u_{n}\left(1 / c ; e_{j}\right) \\
\quad-\beta_{j} u_{n}[1 / c ; \dot{p}(0)] \mid
\end{aligned}
$$

when computing over a finite range of $n$ and $j$ values. In particular, we considered $n \in\{-20, \ldots, 20\}$ and $j \in\{-10, \ldots, 10\}$.

\section{Propagation of phase mixtures: Two-front solutions}

Using the single-front solutions, it is easy to construct two-front solutions that can be compared to the ones we have seen in numerical simulations. Indeed, assuming that, as in the numerical results, the two fronts are symmetric about $n$ $=n_{0}$ (without loss of generality, one can set $\left.n_{0}=0\right)$, one can see that the two-front solutions satisfy

$$
\begin{gathered}
\dot{y}_{n}=-\alpha\left(z_{n}-2 y_{n}+z_{n-1}\right)+\sigma_{a}-y_{n}+\theta(c t+m-|n|), \\
\dot{z}_{n}=-\alpha\left(y_{n}-2 z_{n}+y_{n+1}\right)+\sigma_{a}-z_{n} .
\end{gathered}
$$

Here $n= \pm m$ mark the positions of the fronts at $t=0$ or $c$ $=0$. At $n \geq 0$, the equations coincide with Eq. (16) for a single front (up to translation by a constant), and from symmetry one can see that $y_{-n}(t)=y_{n}(t)$ and $z_{-n}(t)=z_{n-1}(t)$ for $n$ $\geq 0$. Recalling the single-front solution [Eqs. (17), (25), and (26)], we obtain

$$
\begin{gathered}
y_{n}=\sigma_{a}+\phi(|n|-m-c t), \\
z_{n}= \begin{cases}\sigma_{a}+\psi(n-m-c t) & n \geq 0 \\
\sigma_{a}+\psi(-n-1-m-c t) & n<0 .\end{cases}
\end{gathered}
$$

Recall that the actual velocity of the propagating phase mixture is $V=2 c$. The kinetic relation for the two-front solutions is the same as in the single-front case and given by Eq. (32).

The above family of solutions describes the propagation of phase mixture inside phase I (the low-strain phase). Using the symmetry properties of Eq. (36), one can also construct solutions describing the propagation of phase mixture inside phase II. These solutions satisfy

$$
\begin{gathered}
\dot{y}_{n}=-\alpha\left(z_{n}-2 y_{n}+z_{n-1}\right)+\sigma_{a}-y_{n}, \\
\dot{z}_{n}=-\alpha\left(y_{n}-2 z_{n}+y_{n+1}\right)+\sigma_{a}-z_{n} \\
+\theta(n-m-c t-1)+\theta(-n-m-c t-2),
\end{gathered}
$$

with the boundary conditions $y_{n}, z_{n} \rightarrow \sigma_{a}+1$ as $n \rightarrow \pm \infty$, where $\sigma_{a}+1>w_{c}$, so that the limiting strains are in phase II. We obtain

$$
\begin{gathered}
y_{n}=\sigma_{a}+1-\psi(|n|-1-m-c t), \\
z_{n}= \begin{cases}\sigma_{a}+1-\phi(n-m-c t), & n \geq 0, \\
\sigma_{a}+1-\phi(-n-1-m-c t), & n<0 .\end{cases}
\end{gathered}
$$

Remark 1. On might expect that stability of the one-front solutions implies the stability of these two-front solutions, and while numerically the two-front solutions appear stable, a rigorous proof of stability is beyond the scope of this work. In fact, the two-front solutions are roughly a linear combination of two one-front solutions moving in the opposite directions once the applied load is subtracted. An approach to determining the stability of such solutions may be to follow the work of Wright. ${ }^{29}$ However, the context considered in Ref. 29 is different. What is considered are continuum equations that support stable pulses, and the results obtained are in showing the stability of two pulses moving apart.

\section{Trapped equilibrium states and dynamics near the depinning threshold}

At $c=0$, the dynamical system [Eq. (36)] reduces to the difference equations. After changing the variables to $\Phi_{n}$ $=y_{n}-\sigma_{a}$ and $\Psi_{n}=z_{n}-\sigma_{a}$, we obtain

$$
\begin{gathered}
\alpha\left(\Psi_{n}-2 \Phi_{n}+\Psi_{n-1}\right)+\Phi_{n}=\theta(m-|n|), \\
\alpha\left(\Phi_{n}-2 \Psi_{n}+\Phi_{n+1}\right)+\Psi_{n}=0 .
\end{gathered}
$$

Applying discrete Fourier transform

$$
\hat{\Phi}(k)=\sum_{n=-\infty}^{\infty} \Phi_{n} e^{-i n k}
$$

to Eq. (40), followed by inverse discrete Fourier transform, we obtain

$$
\begin{gathered}
\Phi_{n}=\frac{1-2 \alpha}{2 \pi} \int_{\Gamma_{0}} \frac{\sin [(m+1 / 2) k] e^{i n k} d k}{\sin (k / 2) \mathcal{L}_{0}(k)}, \\
\Psi_{n}=-\frac{\alpha}{\pi} \int_{\Gamma_{0}} \frac{\cos (k / 2) \sin [(m+1 / 2) k] e^{i k(n+1 / 2)} d k}{\sin (k / 2) \mathcal{L}_{0}(k)} .
\end{gathered}
$$

Here

$$
\mathcal{L}_{0}(k) \equiv \mathcal{L}(k, 0)=(2 \alpha-1)^{2}-4 \alpha^{2} \cos ^{2}(k / 2)
$$

and $\Gamma_{0}$ is the contour in the complex plane that goes along the interval $[-\pi, \pi]$ on the real line everywhere except near $k=0$ where it goes above the real line. Closing the contour by a rectangle of height that tends to infinity, applying the residue theorem and recalling that the even-numbered and oddnumbered strains are given by $y_{n}=\Phi_{n}+\sigma_{a}$ and $z_{n}=\Psi_{n}+\sigma_{a}$, respectively, we obtain

$$
\begin{aligned}
y_{n}= & \sigma_{a} \\
& + \begin{cases}\frac{1-2 \alpha}{1-4 \alpha}-\frac{(1-2 \alpha) e^{-(m+1 / 2) \rho}}{2 \alpha^{2} \sinh (\rho / 2) \sinh \rho} \cosh (\rho n), & |n| \leq m \\
\frac{(1-2 \alpha) \sinh [(m+1 / 2) \rho]}{2 \alpha^{2} \sinh (\rho / 2) \sinh \rho} e^{-\rho|n|}, & |n| \geq m+1 .\end{cases}
\end{aligned}
$$




$$
z_{n}=\sigma_{a}+ \begin{cases}-\frac{2 \alpha}{1-4 \alpha}+\frac{e^{-(m+1 / 2) \rho}}{2 \alpha \sinh ^{2}(\rho / 2)} \cosh [\rho(n+1 / 2)], & |n+1 / 2|<m \\ -\frac{\sinh [(m+1 / 2) \rho]}{2 \alpha \sinh ^{2}(\rho / 2)} e^{-\rho|n+1 / 2|}, & |n+1 / 2|>m .\end{cases}
$$

Here we used the fact that the roots of $\mathcal{L}_{0}(k)=0$ inside the strip $-\pi \leq \operatorname{Re} k \leq \pi$ are $k= \pm i \rho$, with $\rho$ defined in Eq. (30).

The constraints that $y_{n} \leq w_{c}$ for $|n| \geq m+1$ and $y_{n} \geq w_{c}$ for $n \leq m$, assumed when the equilibrium Eq. (40) was obtained, result in certain bounds on the applied stress $\sigma_{a}$. Due to monotonicity of obtained equilibrium solutions $y_{n}$ at $n \geq 0$ and symmetry about $n=0$, we only need to require $y_{m} \geq w_{c}$ and $y_{m+1} \leq w_{c}$. This gives the lower and upper bounds for the trapping region in which the constructed equilibrium states exist,

$$
w_{c}+\frac{(1-2 \alpha) e^{-\rho(m+1 / 2)} \cosh (\rho m)}{2 \alpha^{2} \sinh (\rho / 2) \sinh \rho}-\frac{1-2 \alpha}{1-4 \alpha} \leq \sigma_{a} \leq \sigma_{a}^{*}(m),
$$

where we defined

$$
\sigma_{a}^{*}(m)=w_{c}-\frac{(1-2 \alpha) e^{-\rho / 2}\left(1-e^{-(2 m+1) \rho}\right)}{4 \alpha^{2} \sinh (\rho / 2) \sinh \rho} .
$$

If the strict inequalities hold, the equilibria are stable, meaning that they are local minimizers of the free energy with respect to variations that have zero average strain and do not introduce new phase boundaries. Indeed, in this case each spring remains in one of the two convex phase regions and this, together with the inequality $0<\alpha<1 / 4$, implies that the energy difference is a positive-definite quadratic form. ${ }^{27}$ The upper stability bound $\sigma_{a}^{*}(m)$ is the value of $\sigma_{a}$ at which the strain $w_{2 m+2}=y_{m+1}$ in the $(2 m+2)$ th spring reaches the critical strain, setting the front in motion.

Setting $m=0$ in Eqs. (42) and (43) and recalling that $w_{2 n}=y_{n}$ and $w_{2 n+1}=z_{n}$, we obtain the equilibrium solution with a single spring in phase II that was observed in numerical simulations,

$$
w_{n}=\frac{\cos (\pi n) e^{-\rho|n| / 2}}{2 \alpha \sinh (\rho / 2)}+\sigma_{a} .
$$

The upper stability threshold for this solution is

$$
\sigma_{a}^{t r}=\sigma_{a}^{*}(0)=w_{c}-\frac{e^{-\rho}}{2 \alpha \sinh (\rho / 2)} .
$$

This is the threshold applied stress above which the equilibrium state is no longer stable and the phase mixtures start forming and propagating.

Equilibria with $m>0$ are also observed numerically if the initial condition has even-numbered springs with $|n| \leq 2 m$ at critical strain. The upper bound for these solutions, defined in Eq. (45), decreases as $m$ grows, reaching the value

$$
\sigma_{a}^{\infty}=\lim _{m \rightarrow \infty} \sigma_{a}^{*}(m)=w_{c}-\frac{(1-2 \alpha) e^{-\rho / 2}}{4 \alpha^{2} \sinh (\rho / 2) \sinh \rho}
$$

in the limit of infinite $m$.

Consider now the two-front dynamic solution with $m=0$ and small $V>0$. For example, the functions $\phi(\xi)$ and $\psi(\xi)$ for the case $V=0.02$ are shown in Fig. 7. Recalling that $w_{2 n}(t)=y_{n}(t)$ and $w_{2 n+1}(t)=z_{n}(t)$, with the even and odd strains given by Eq. (37), one can see that the small-velocity solution describes a stick-slip dynamic regime. As we increase $t$ from zero (or, equivalently, decrease $\xi$ for a given $n$ ), the fast dynamic regime (slip) brings the strains to constant values near an equilibrium state with $m=0$, where the solution remains for the remainder of the period $T=1 / V$ (stick regime). Following another fast dynamic regime, the solution approaches the next equilibrium state $(m=1)$, and so on. Figure 8 depicts solutions during the first four stick regimes. Note that in each near-equilibrium state the two evennumbered springs near the boundaries of the phase mixture have strains close to the $w_{c}$, triggering the next transition. At smaller $V$, each stick regimes lasts longer, and the dynamic solution is closer to an equilibrium state during it, with $m$ tending to infinity as $t$ increases.

Hence $\sigma_{a}^{\infty}$ is the bifurcation point from which the branch of dynamic solutions emerges. Note that it is strictly less than $\sigma_{a}^{t r}$, the upper limit of the trapping region of $m=0$ equilibrium. This implies that at $\sigma_{a}$ between the two threshold values the equilibrium state coexists with the steady propagation of the phase mixture.

\section{E. Kinetic relation}

A typical kinetic relation $\bar{\sigma}(V)$ is depicted in Fig. 9. The thick segment at $V=0$ depicts the trapped equilibria constructed in Sec. IV D, and the thin line show the kinetic relation for the dynamic solutions obtained in Sec. IV C. We

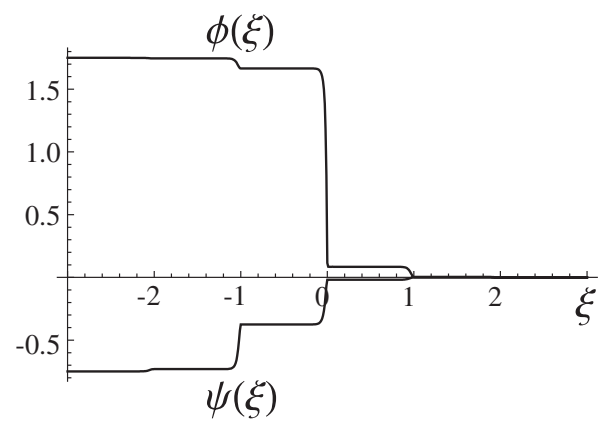

FIG. 7. The functions $\phi(\xi)$ and $\psi(\xi)$ for solution at $V=0.02$. Other parameters: $\alpha=0.15, w_{c}=1$. 

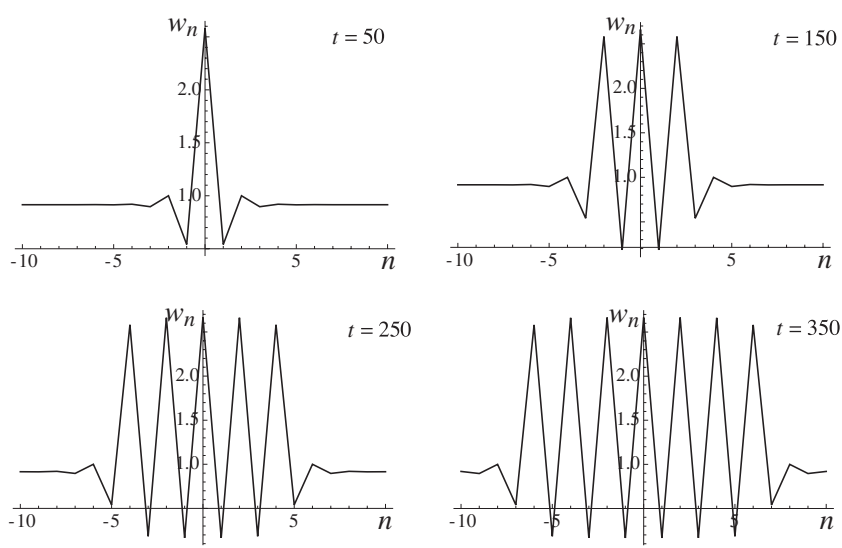

FIG. 8. Near-equilibrium strain profiles at $V=0.02$. Other parameters: $\alpha=0.15, w_{c}=1$.

now consider two limits of the kinetic relation,

a Limit $V \rightarrow \infty$. Note that as $V$ tends to infinity, $\bar{\sigma} \rightarrow 1 / 2$ for any $\alpha$. This can be seen by observing that at large $V$ the roots in $M^{+}(V / 2)$ tend to infinity, so that the sum in the second equality in Eq. (32) tends to zero. Physically, this means that there are no solutions with steadily propagating phase mixtures above the spinodal stress $\sigma_{a}=w_{c}$, or $\bar{\sigma}=1 / 2$.

b. Limit $V \rightarrow 0$. As we already remarked above, in the limit $V \rightarrow 0$ the dynamic solutions approach the bifurcation point $\bar{\sigma}(0)=\sigma_{a}^{\infty}-\sigma_{M}$. Since $\bar{\sigma}(0)<1 / 2$, the dynamic solutions coexist with equilibrium ones when $\bar{\sigma}$ is between these two thresholds.

\section{F. Comparison of numerical and analytical solutions}

Figure 9 compares the kinetic relation generated by the analytical solution (solid line) to the numerical values of $V$ at a given $\bar{\sigma}$ (dots). Recall that the actual kinetic relation (shown by a solid line in Fig. 9) consists of trapped equilibria and the analytical curve for nonzero velocity of front propagation. Numerical simulations suggest stability of analytical solutions along this curve. For $\sigma$ below the trapping threshold, numerical solution with initial data obtained from the analytical solution agrees with it at larger times.

A direct comparison of the strain profiles zoomed in around the right front of the moving phase mixture is shown in Fig. 10. One can see that the agreement is remarkably good, suggesting that the analytical solution we obtained is stable. (a)

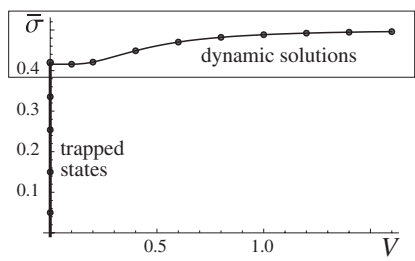

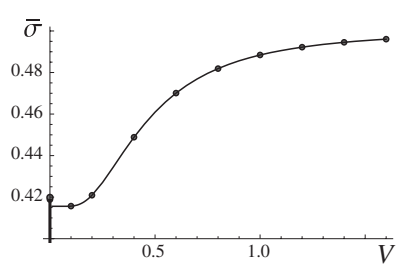

FIG. 9. (a) The kinetic relation $\bar{\sigma}(V)$ at $\alpha=0.15$ for the constructed trapped equilibrium states and dynamic solutions. (b) The enlarged picture of the area inside the rectangle in (a). The numerical solutions are shown by dots.
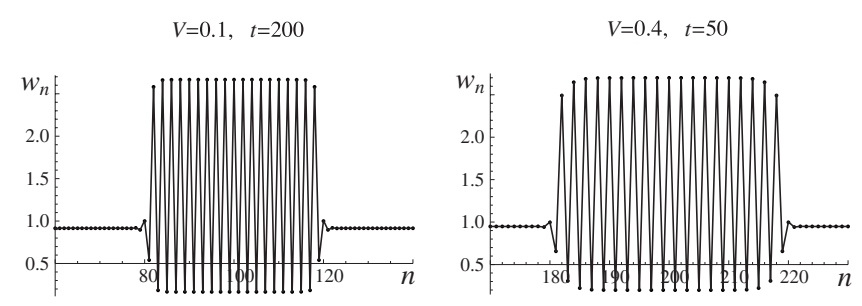

FIG. 10. Snapshots of strain profiles generated by the numerical simulation (dots) and analytical solution (lines) at $\alpha=0.15$ and $w_{c}$ $=1$. In each case the analytical solution was shifted so that the center of symmetry coincides with that of the numerical solution. The profile at $V=0.1$ was obtained using the analytical solution $w_{n}(100)$ as initial data, while the $V=0.4$ profile evolved from the initial data described in Sec. III.

\section{NUMERICAL SIMULATIONS FOR TRILINEAR AND CUBIC STRESS-STRAIN LAWS}

The bilinear nonlinearity allowed us to construct exact solutions for the propagating phase mixtures. However, the question remains whether some of the results are due to the degeneracy of the model and whether a similar steady propagation of phase mixtures also takes place when the bilinear stress-strain law is replaced by a continuous curve. We conclude the paper by investigating this numerically.

We start by considering a trilinear stress-strain law

$$
\sigma(w)= \begin{cases}w, & w \leq w_{1} \\ \frac{\left(w_{1}-w_{2}+1\right) w-w_{1}}{w_{1}-w_{2}}, & w_{1} \leq w \leq w_{2} \\ w-1, & w \geq w_{2},\end{cases}
$$

which, in addition to linear regions of phase I $\left(w \leq w_{1}\right)$ and phase II $\left(w \geq w_{2}\right)$, features a nontrivial spinodal region $w_{1}$ $\leq w \leq w_{2}$, where the slope is negative. A chain deformed uniformly with the strain in this region is unstable. In the limit $w_{1} \rightarrow w_{2}$ we recover the bilinear law used above.

Solving Eq. (11) numerically with $\sigma(w)$ given by Eq. (49) (with $\alpha=0.15, w_{1}=0.8$, and $w_{2}=1.2$ ) and the initial conditions as described in Sec. III, we obtain the following results.
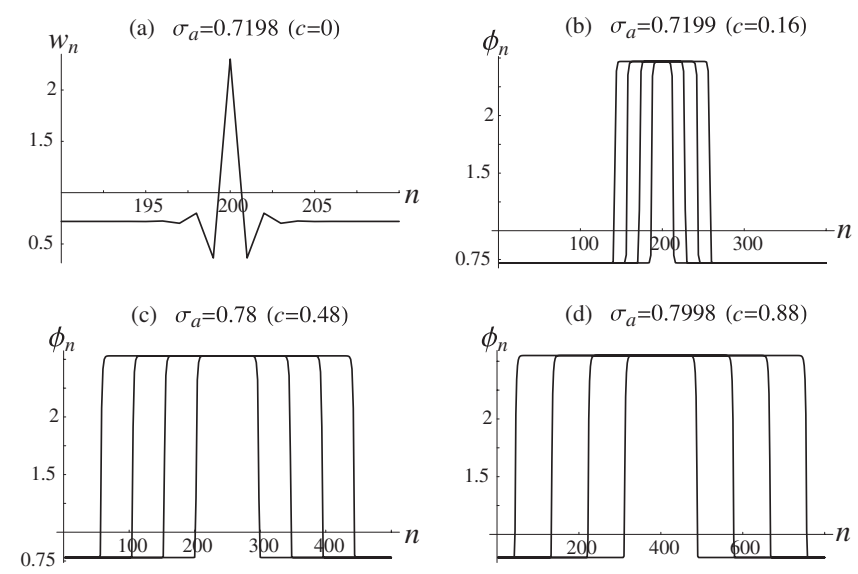

FIG. 11. (a) Stationary strain profile for trilinear material at $\sigma_{a}$ below the trapping threshold. [(b)-(d)] Profiles of $\phi_{n}(t)=w_{2 n}(t)$ $-\sigma_{a}$ at $t=100,200,300$, and 400. Here $w_{1}=0.8, w_{2}=1.2, \alpha=0.15$. 


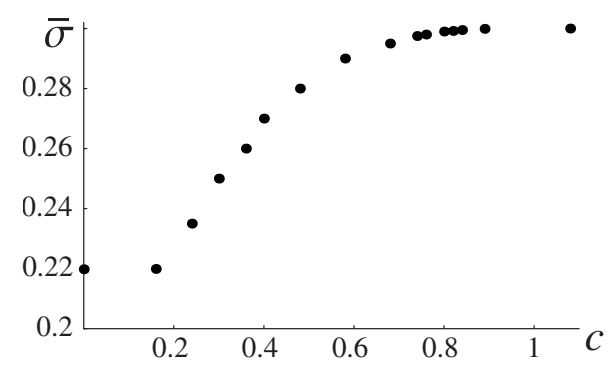

FIG. 12. Kinetic relation $\bar{\sigma}(c)$ for the steadily propagating phase mixtures in a trilinear material. Here $w_{1}=0.8, w_{2}=1.2, \alpha=0.15$.

At $\sigma_{a} \leq 0.7198$ the simulation yields a trapped equilibrium state similar to the ones observed with bilinear law [see Fig. 11(a)]. At larger stress that does not exceed the spinodal value $\sigma_{S}=w_{1} \quad\left(0.7198 \leq \sigma_{a} \leq 0.8\right)$ we observe the steady propagation of the phase mixture. This can be seen in Figs. $11(\mathrm{~b})-11(\mathrm{~d})$, where only the profiles of $\phi_{n}(t)=w_{2 n}(t)-\sigma_{a}$ are depicted, in order to show front propagation at different times on the same plot. The kinetic relation $\bar{\sigma}(c)$ for the dynamic solutions is shown in Fig. 12. As before, we define $\bar{\sigma}=\sigma_{a}-\sigma_{M}$ as the difference between applied and Maxwell stresses. The Maxwell stress $\sigma_{M}$ for the trilinear material is the same as in the bilinear case (in the figure $\sigma_{M}=0.5$ ). One can see that as in the bilinear case, the kinetic curve levels off at the spinodal stress $\sigma_{S}=0.8$ (or $\bar{\sigma}=0.3$ ). Numerical simulations suggest stability of all dynamic solutions shown.

At applied stress above the spinodal value and below a certain threshold, $0.8<\sigma_{a}<0.836$, the numerical simulations yield static solutions with a phase mixture in the middle and phase II elsewhere; see Fig. 13. As the applied load is increased, the length of the phase mixture interval decreases, and at sufficiently high applied load, $\sigma_{a} \geq 0.836$, constantstrain equilibria are observed. Similar solutions, given by the $c \rightarrow 0$ limit of Eq. (39), can also be obtained in the bilinear case if the initial perturbation from the constant-strain equilibrium is sufficiently strong. In the trilinear case they are seen even with a small initial perturbation because of the stronger spinodal instability. law,

We now consider the fully nonlinear cubic stress-strain

$$
\sigma(w)=w(w-1 / 2)(w-1)
$$

that has two phase regions $\left(\sigma^{\prime}(w)>0\right), w<w_{1}=(3-\sqrt{3}) / 6$ (phase I) and $w>w_{2}=(3+\sqrt{3}) / 6$ (phase II), and spinodal region in between. The results of numerical simulations for this
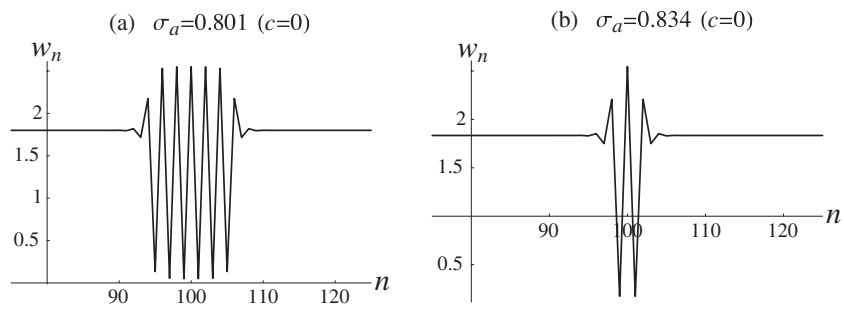

FIG. 13. Static solutions above the spinodal stress $\sigma_{S}$. Here $w_{1}=0.8, w_{2}=1.2, \alpha=0.15$.
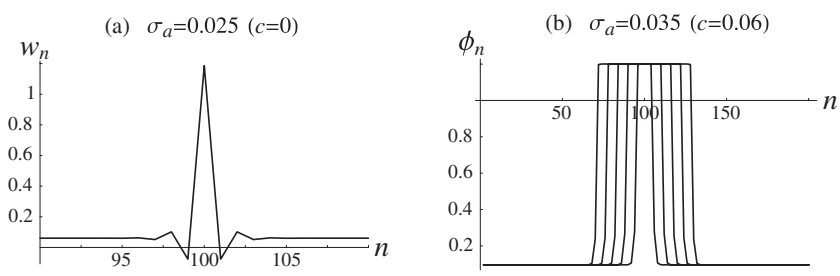

(c) $\sigma_{a}=0.04(c=0.1)$

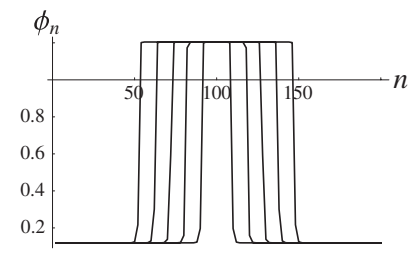

(d) $\sigma_{a}=0.045(c=0.14)$
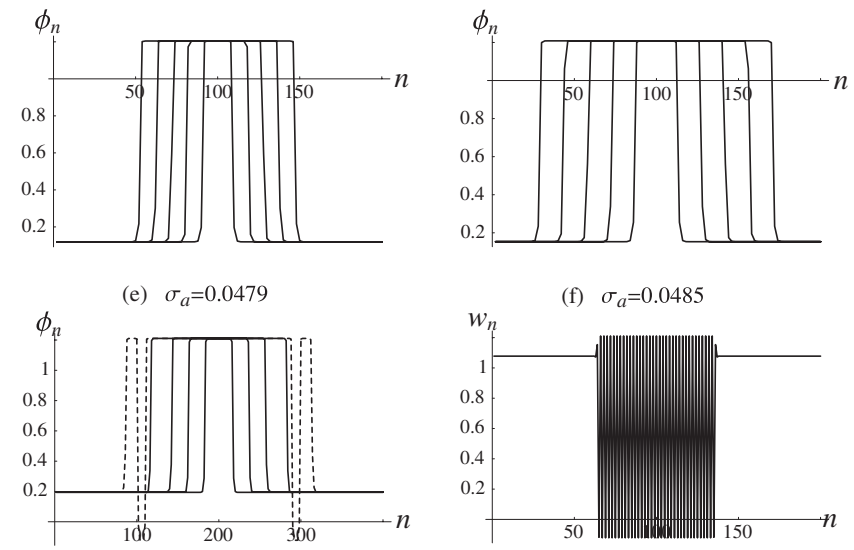

FIG. 14. (a) Stationary strain profile for cubic material at $\sigma_{a}$ below the trapping threshold. [(b)-(e)] Profiles of $\phi_{n}(t)=w_{2 n}(t)$ $-\sigma_{a}$ at $t=100,200,300,400$, and 500. Here $\alpha=0.05$. (f) Stationary strain profile above the spinodal stress.

fully nonlinear case are shown in Fig. 14. An equilibrium state similar to those seen in bilinear and trilinear cases is observed when the applied stress is below a trapping threshold, $\sigma_{a}<0.03$; see Fig. 14(a). At stresses above the trapping threshold and below a certain value we see a stable steady propagation of phase mixtures, as shown in Figs. 14(b)-14(d) [once again, we only show $\phi_{n}(t)$ profiles for clarity].

However, stresses sufficiently close to but below the spinodal stress $\sigma_{S}=0.0481$ result in an instability of this steady motion, as illustrated in Fig. 14(e). Specifically, the fronts propagate steadily for some time (at least until $t=300$ ), but then they speed up $(t=400)$, and soon after a pair of new fronts is nucleated on each side (see the dashed curve at $t$ $=500$ ). Subsequently, the old fronts stop moving, while the new ones propagate. At larger $\sigma_{a} \leq \sigma_{S}$, more new fronts nucleate. These results indicate instability of the steady motion for stresses near the spinodal value for the fully nonlinear model. Interestingly, such instability was not observed in the bilinear and trilinear cases.

At stresses above the spinodal value we see the equilibrium states with phase mixtures [Fig. 14(f)] that are similar to those observed in the trilinear case, followed by constantstrain equilibrium at higher applied stresses.

\section{CONCLUDING REMARKS}

In this paper we used a discrete model with bilinear nearest-neighbor interactions and harmonic next-to-nearest neighbors to construct one-front and two-front analytical solutions describing steady propagation of phase mixtures 
though a one-dimensional lattice and derive the kinetic relation between the applied stress and the velocity of the mixture. At small velocities, the motion is of stick-slip type, and as velocity tends to zero, the driving force approaches the pinning threshold below which there is no steady propagation, and the phase mixtures are trapped in one of the multiple equilibrium configurations. The trapping region where the equilibrium solutions exist goes beyond the pinning threshold, so that solutions with trapped and slowly moving interfaces coexist. Stability of the constructed two-front solutions is suggested by the results of the numerical simulations of the initial value problem, in which solutions converged to the traveling-wave-like propagation constructed analytically. To verify the stability of one-front solutions we employed the approach of Ref. 28 to obtain a stability condition which was then checked numerically.

We also conducted numerical simulations for trilinear and cubic nearest-neighbor interactions. In both cases steady propagation of two-front phase mixtures similar to the one we constructed was observed and appeared stable within a certain range of applied loads. In the case of cubic interactions the motion becomes unstable at near-spinodal values of the applied stress when the spinodal instability leads to the nucleation and motion of new fronts.

The approach we used to construct the analytical solutions, seeking traveling-wave solutions for odd-numbered and even-numbered strain variables, can be extended to obtain other periodic solutions on lattices, including those that describe dynamics of commensurate-incommensurate phase boundaries in a chain that includes competing long-range interactions. Future work will also include considering the underdamped dynamics and higher-dimensional lattices and further investigating the effects of full nonlinearity.

\section{ACKNOWLEDGMENTS}

This work was supported in part under NSF Grants No. DMS-0443928 (A.V.), No. DMS-0513438, and No. DMS0812800 (E.S.V.V.).

\footnotetext{
*aav4@pitt.edu

†evanvleck@math.ku.edu

${ }^{1} \mathrm{~K}$. Bhattacharya, Microstructure of Martensite-Why it Forms and How it Gives rise to the Shape Memory Effect (Oxford University Press, Oxford, 2003).

${ }^{2}$ C. Chu, Ph.D. thesis, University of Minnesota, 1993.

${ }^{3}$ J. Ball, P. Holmes, R. James, R. Pego, and P. Swart, J. Nonlinear Sci. 1, 17 (1991).

${ }^{4}$ G. Friesecke and J. McLeod, Arch. Ration. Mech. Anal. 133, 199 (1996).

${ }^{5}$ A. Vainchtein, J. Elast. 57, 243 (1999).

${ }^{6}$ J. M. Ball and R. D. James, Arch. Ration. Mech. Anal. 100, 13 (1987).

${ }^{7}$ J. M. Ball and R. D. James, Philos. Trans. R. Soc. London, Ser. A 338, 389 (1992).

${ }^{8}$ S. Müller, Calculus Var. Partial Differ. Equ. 1, 169 (1993).

${ }^{9}$ A. Vainchtein, T. Healey, P. Rosakis, and L. Truskinovsky, Physica D 115, 29 (1998).

${ }^{10}$ A. Vainchtein, T. Healey, and P. Rosakis, Comput. Methods Appl. Mech. Eng. 170, 407 (1999).

${ }^{11}$ L. Truskinovsky and G. Zanzotto, J. Mech. Phys. Solids 44, 1371 (1996).

${ }^{12}$ L. Truskinovsky and A. Vainchtein, Continuum Mech. Thermodyn. 20, 97 (2008).

${ }^{13}$ S. Pagano and S. Paroni, Q. Appl. Math. 61, 89 (2003).
}

${ }^{14}$ H. P. McKean, Adv. Math. 4, 209 (1970).

${ }^{15}$ J. Rinzel and J. B. Keller, Biophys. J. 13, 1313 (1973).

${ }^{16}$ O. M. Braun and Y. S. Kivshar, The Frenkel-Kontorova Model: Concepts, Methods and Applications (Springer-Verlag, Berlin, Heidelberg, 2004).

${ }^{17}$ A. Carpio and L. L. Bonilla, SIAM J. Appl. Math. 63, 1056 (2003).

${ }^{18}$ G. Fáth, Physica D 116, 176 (1998).

${ }^{19}$ J. P. Keener, SIAM J. Appl. Math. 47, 556 (1987).

${ }^{20}$ J. Cahn, J. Mallet-Paret, and E. S. Van Vleck, SIAM J. Appl. Math. 59, 455 (1998).

${ }^{21}$ S. N. Chow, J. Mallet-Paret, and E. S. Van Vleck, Random Comput. Dyn. 4, 109 (1996).

22 J. W. Cahn, S. N. Chow, and E. S. Van Vleck, Rocky Mt. J. Math. 25, 87 (1995).

${ }^{23}$ J. W. Cahn and E. S. Van Vleck, Acta Mater. 47, 4627 (1999).

${ }^{24}$ J. P. Sethna, Entropy, Order Parameters and Complexity (Oxford University Press, New York, 2006).

${ }^{25}$ P. Bak, Rep. Prog. Phys. 45, 587 (1982).

${ }^{26}$ T. Janssen and A. Janner, Adv. Phys. 36, 519 (1987).

${ }^{27}$ L. Truskinovsky and A. Vainchtein, J. Mech. Phys. Solids 52 , 1421 (2004).

${ }^{28}$ S. N. Chow, J. Mallet-Paret, and W. Shen, J. Dyn. Differ. Equ. 149, 248 (1998).

${ }^{29}$ J. D. Wright, J. Dyn. Differ. Equ. (to be published). 